\title{
Multi Domain Matrix (MDM) of the Vertical Hydrogenerator
}

\author{
Ivan TRIPLAT, Dorian MARJANOVIĆ, Lucijan STAMAĆ
}

\begin{abstract}
Purpose of this work was to show connections between vertical hydrogenerator design parameters. Authors made DSM (Design Structure Matrix), to show design parameters dependencies, first matrix size was reduced and it does not consist of all design parameters, it consists only of more relevant design parameters to be solvable. Final size of matrix is $221 \times 221$. Matrix shows that electrical generator in some ideal conditions could have almost straightforward path during design procedure. DSM matrix is still in process of verification. After finishing DSM of design parameters, authors made MDM (Multi Domain Matrix) which connects design parameters with mechanical calculations and generator main drawings. This matrix has the purpose to help managers, electrical design experts, other design experts and any others who should not need knowledge about generator design process to easily track changes between parameters and to see how change of some parameter affects drawings and calculations. At the end authors made VBA program which visually shows connections.
\end{abstract}

Keywords: design parameters; Design Structure Matrix (DSM); Multi Domain Matrix (MDM); vertical hydrogenerator

\section{INTRODUCTION}

The large vertical hydrogenerator is a comprehensive product composed of several thousand parts. Product development and the design process of a product at such complexity demands multidisciplinary design approach because it integrates knowledge from three major domains: electrical, mechanical and thermal. Considering function and structure the electric generator is a well-known product with the primary purpose to deliver electric current through the foreseen lifetime sustainably. Based on functional requirements the generator design process starts with the determination of the main active parts: the diameters of the stator core, the diameter of rotor poles, number of poles, number of stator slots, etc. With generator's main dimensions defined, design engineers will have to fit generator's subassemblies and components: stator housing, brackets, bearings, fans and all other components, to the existing or new foundations. Necessary stress, strain and dynamic calculations are performed in order to satisfy the design life cycle $(30-40$ years of operation depending on customer requirements, and recently they demand 80 years of service). Particular attention is given to the rotating parts due to the dynamics and vibrations. Fans, poles, and ventilation channels are calculated considering and predicting ventilation and thermal conditions to achieve normal temperatures in all operation regimes.

Due to the complexity of the problem, and ever increased requirements the optimisation of the design process and the product being designed is imperative. The pragmatic call for partial suboptimisation seems awarding but is often a source of rework, because optimising one problem, like optimisation of mechanical calculations stream, will not bring to the optimal solution of the overall design process [12].

To increase the generator design efficiency, it is of critical value to utilise the company's know-how, the experience and knowledge captured through many previous designs to enhance the new products. A system that will enable a structured and systematic insight into a generator design knowledge, an organisation allowing knowledge capture and reuse is especially essential for the novel designers as well as for the experienced designers.
The goal of this work was to explore possibilities to improve the design process of electrical generator considering the process duration [2] enabling better management of future design projects and minimising the reworks that occur as a consequence of non-updated parameters or not-updated drawings and calculations. This situation arises as a combination of product complexity and human errors due to the lack of experience. Thus improving the change management capabilities is also a goal of the research presented in this paper.

In addition, it would be useful to make a visual representation of the design process to help manage the problem [6].

To achieve goals, design structure matrix DSM of main generator design parameters was developed incorporating all the parameters starting with electrical design, and propagating in other domains of design.

The Design Structure Matrix (DSM), somewhere also referenced as dependency structure matrix, is used to analyse and represent relations regarding generator structure, design process and the system-level design knowledge. Well-presented knowledge captured through DSM could improve design process enabling better insight into design history, design routine thus allowing better understanding of design rationales, relationships and hidden dependencies. It could also help to predict changes in adaptation and redesign of existing generators as well as to reuse current solutions in the new design tasks.

\subsection{Hydrogenerator Design Structure}

It is well known that vertical hydrogenerator of high power incorporates more than thousands of components, and because of that one of possibilities to manage design parameters is to take more relevant design parameters and to represent them in a matrix form (DSM). The DSM has been chosen due to advantages of simplicity and conciseness in representation [4]. Supported by appropriate tools, the designer can utilise DSM to identify patterns in system architectures, such as modules and cycles.

For the sake of readability, a brief description of main hydrogenerator design indicating structure and main components is included. On the top level structure of 
electrical generator, two main groups of components (subassemblies and parts) could be identified: the rotating components and the stationary components as illustrated in Fig. 1. Generator parts on which this work relates are bearings collars, generator shaft, rotor spider, rotor rim, poles, fans, brackets, stator housing, stator core, stator winding and bearings.

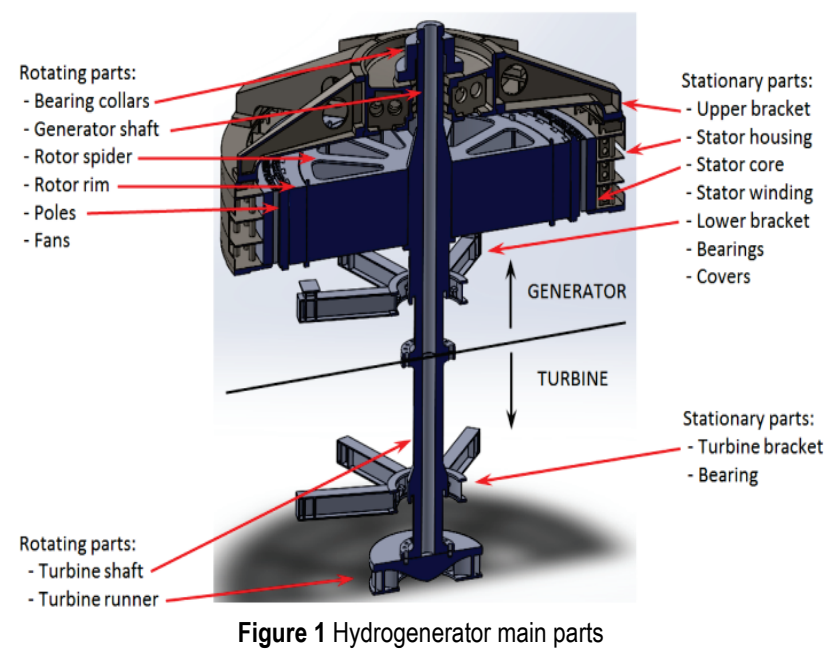

During the design process, generator usually has 17 main drawings which are below generator assembly drawing and 19 main calculations. All main drawings are depicted in Fig. 2 while Fig. 3 depicts main calculations.

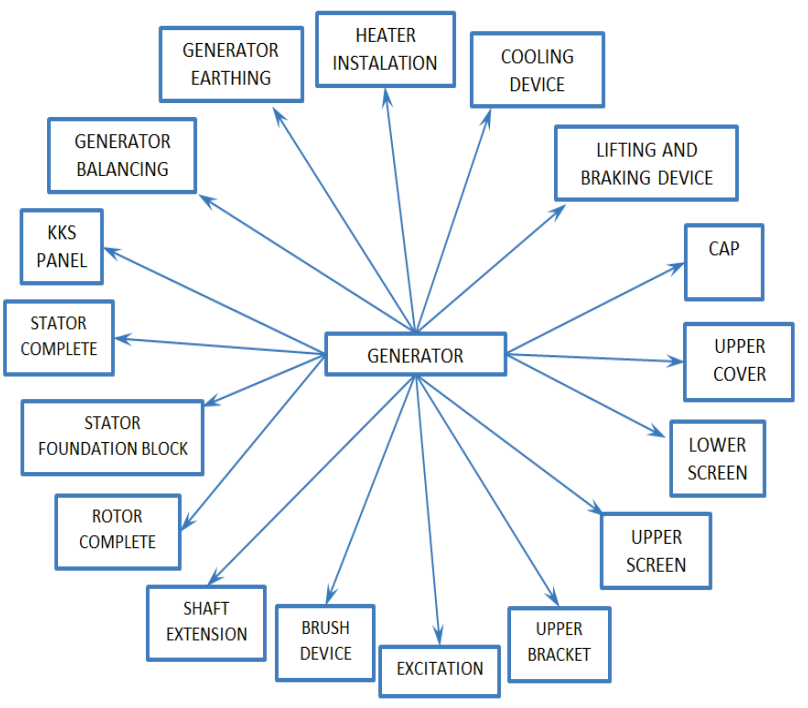

Figure 2 Hydrogenerator main drawings

To analyse vertical generator design parameters stream, we applied DSM (Design Structure Matrix) method which is developed by Steward in 1970, who used a matrix to show relationships in a product. Although Steward came up with matrix method many years ago, practical, industrial application came up many years later in NASA, Boeing, General Motors, Intel etc. Up to now, DSM is widespread in the industry for representation of static relationships. DSM shows information flow in a design process, and it says what optimal stream is in particular design stage.

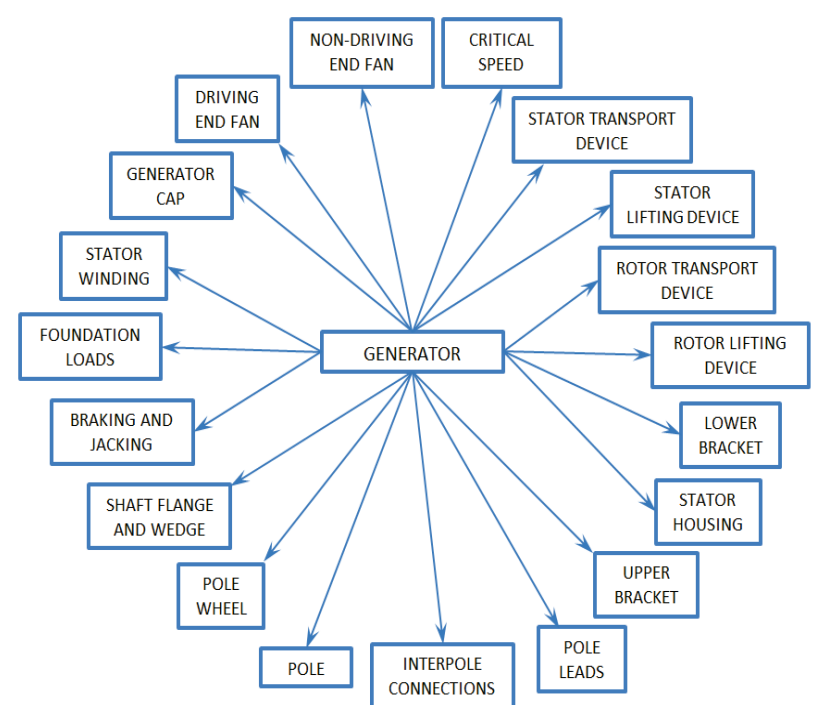

Figure 3 Calculations during design process

This paper presents the DSM development of generators' main mechanical calculations, like calculation of stator core, calculation of stator housing, calculation of upper and lower brackets, calculation of foundation loads, critical speed calculation etc. The product DSM developed models relations between components, brackets, shaft, rotor spider, rotor rim, poles, fans, stator core, stator housing, bearings, etc. which give insight in only one part of generator design process. DSM increases architectural understanding, identifies modules, predicts system interaction, reduces process duration, reduces rework, improves organisation etc., but DSM-s done so far couldn't show all interconnections between domains in a design process.

This DSM incorporates only more relevant design parameters, to reduce the number of parameters to the acceptable level, final DSM is a matrix of size $221 \times 221$. DSM should improve generator design process time, and minimise unnecessary reworks. After partitioning of parameters, the goal was to connect all parameters with calculations and drawings. These should help to manage changes in a design process.

\subsection{State of Art - a Brief Overview of DSM and MDM}

The DSM arose from the work of professor Don Steward in early 1970s. The work was reported in the internal General Electric. The work was not widely known until 1981 when the book [17] and an article [18] were published. The DSM had started with industry applications when MIT researchers used it in NASA, Boeing, Intel, and General Motors in the 1990s [6, 7]. Today DSM is in a wide range of industries with numerous applications, and there are hundreds of papers done so far.

Browning's [2] review identified three types of DSM applications - models of products, organisations, and processes. Browning also indicated a fourth DSM type, a parameter-based DSM, as essentially a high-fidelity process DSM.

In further literature consolidation, DSM models are classified into four types within three main categories [6]. The Product Architecture DSM is representing the static architecture whose elements exist simultaneously. 
Products are engineered artefacts, systems with components which physically interact, one with another or organisations with elements which communicate one with another represented by square matrices. A square matrix was used to describe the product components quite earlier in the works of Simon in early 1960.

Organization Architecture DSMs are also static representations of an organisation. The organisation is considered a group, network of people and teams with a common goal. Viewed as a system an organisation also has an architecture - its structure, "embodied in its people, their relationships to each other and the organisation's environment, and the principles guiding its design and evolution" [7].

The primary focus of static DSM-based representations is to minimise technical complexity by clustering the system components in a way that minimizes the technical interactions between clusters of components, i.e. complex interactions are grouped within clusters [8].

Process Architecture DSMs have received the most attention of all DSM applications. The process is considered as a system of interacting activities executed to achieve a planned project or business function. The Process DSM is used in process design and planning to identify interactions between activities as well as for guiding and evaluation during implementation. The process DSM models will include at least the mappings of activities and input-output relationships between activities. Although it represents a temporal flow in which elements are timedependent, it should be noted that Process DSM could provide only a partial view of the process [3].

The importance of modelling both the domain and between domains relationships led to the development of the multidomain matrix (MDM), which represents multidomain DSM-s represented in a single matrix. In other words multidomain matrix - MDM enables analysis of a system's structure across multiple domains, abridging each single analysis into one DSM that represents multiple domains at a time like organisation architecture, product architecture, or process architecture matrix. The idea of multiple views in system analysis is not new. Eppinger, Salminen and Maurer (2001) reported about three mappings across different DSM domains. DSM has been extended to more than one domain, and later Maurer [13] called it multidomain matrix (MDM). In the recent literature matrix-based approaches integrating multiple views ("domains") become more and more accepted to manage several perspectives onto a system. Although there is no clear recommendations how to perform system analysis with MDM, since MDM might emphasize a mixture of temporal and static DSM [6, 7] MDM is appropriate tool for analysis, especially when it comes to large structures. According to [6, 7], MDM showed good potential for modelling of complex and multi-domain systems. Recent reports describe diverse applications of MDM like: analysis of engineering change requests [1], product development planning [11] or change management in manufacturing systems [16]. The complexity of the system considered in this research analysis in different domains is required as a precondition of the research. Considering inherently multidomain perspectives of this analysis (parameters, processes and technical documentation), choosing the multidomain matrix (MDM) is justified.

The purpose of these MDM is to show all connections which are sometimes hidden and to improve visualization of a process $[19,10]$. These MDM also indicate an optimal order of design parameters, which should later show the optimal order of making calculations and drawings and at the end help improve the design process. For a further study on DSM and MDM literature full list of references the readers are advised to consult the www.dsmweb.org.

This paper used process architecture DSM, other DSM types have not been considered since matrix elements or tasks are design parameters which are time-dependent. In DSM systems, partitioning algorithm reorders columns and rows to move relationships below diagonal and if that is not possible, then moves relationships as close as possible near diagonal. The distance between relations (above diagonal) and diagonal, indicates the number of tasks, that means relations farther away from diagonal makes longer iteration time.

DSM could have dependent, independent, interdependent and conditioned links as shown in Fig. 4 and Fig. 5.

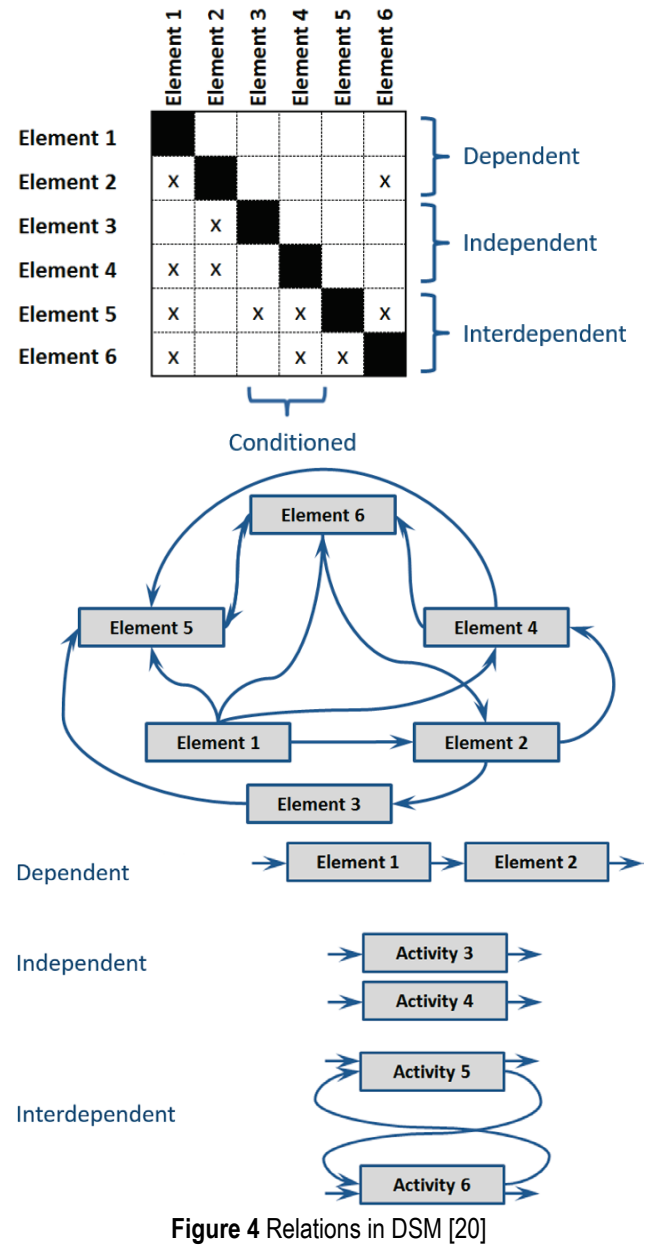

Clustering is applicable to static models like Product architecture and Organization Architecture, while timebased models need to be analysed with sequencing algorithm. After partitioning of DSM, matrix looks like the matrix in Fig. 5 and tasks could be done serial (one after another), parallel or interdependent. 


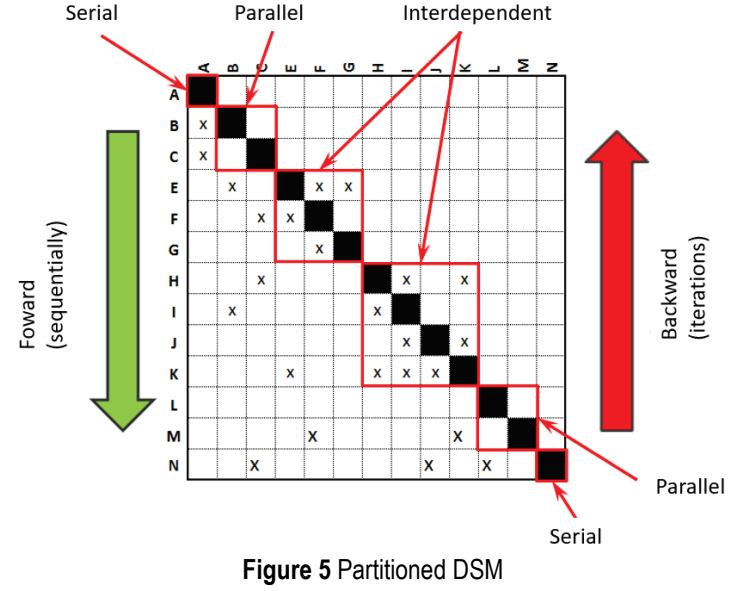

Morelli [15], was first who developed multidomain DSM model, with connections between organisation and process domains. Eppinger, Salminen and Maurer (2001) talked about three mappings across different DSM domains. DSM has been extended to more than one domain, and later Maurer (2007) called it multidomain matrix (MDM). It is not the best way of MDM analysis, since MDM consists of mixture of temporal and static DSM [12]. Eppinger \& Browning said that MDM has good potential for modelling of complex and multi-domain systems.

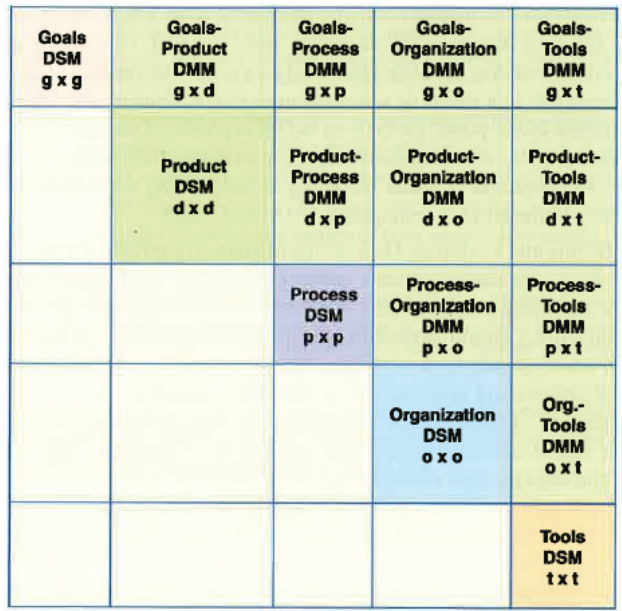

Figure 6 Table of DSM \& DMM, which forms MDM [5]

\subsection{Data Collection}

During the initial stage aggregation of all the design parameters using precession of information flow was conducted. The design parameters denote all the qualitative physical and functional variables of the generator are used or generated during the design process. Design parameters include variables used in electrical design (i.e. generator voltage, power, nominal moment, short circuit moments, nominal speed of rotation, etc.), mechanical design stage (i.e. geometrical data like diameters, heights, and other relevant dimensions) and physical parameters used for calculations during design process (like critical speed, radial stiffness of stator housing and upper bracket, radial stiffness of lower bracket, loads on the upper bracket, loads on the lower bracket, time to stop generator, pressure needed for jacking rotor, etc.).
Such data analysis has not been reported in the literature especially for large electrical generators. The number of parameters included in the analysis should be carefully considered to be manageable with DSM. The reduction of the design parameters resulted with greater readability of the model as well as the calculation time became acceptable. The reduction process was iterative, and the final structure of the most important design parameters included in a DSM were achieved through several meetings with design experts. The initial model was developed considering electrical and mechanical parameters while the others have been added, evaluated and verified in group design meetings in three passes. The first phase was based on the individual work of design engineering experts and electric engineering experts. The tasks have been divided according to the participant's expertise due to the size of a matrix. The matrix of $221 \times 221$ would take too much time for every expert goes through the whole matrix. Initial meeting with all the experts designers in design and development department was conducted to explain how to read the DSM matrix and how to give a suggestion for new/wrong precedence connection between parameters. Every expert was assigned to work on parameters which (s)he covers in everyday work. After collecting of all suggestions and opinions, the matrix was updated with the suggested precedence of relationships.

For the second evaluation of DSM matrix, a verification with a few design experts was conducted. A walk-through of all the matrix to verify the first phase. Although the experts were selected based on their experience, we find out that every expert has his own view on the precedence of relationships for some of the design parameters. The precedences added or altered in second verification circle changed the precedence relationships between parameters done before.

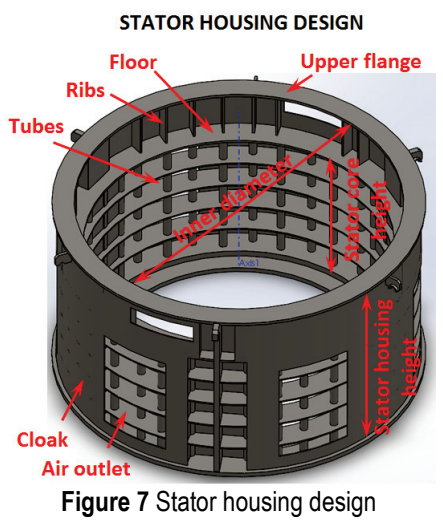

To avoid confusions and to minimise excessive, redundant relations, we decided to do one more, final verification with defined design stream for the most of components. These checks will be done with a few experts (every expert for his part, including electric engineer experts), with additional care to follow some ideal design stream. The final verification is still under way. The design stream as shown in Fig. 7 illustrates the simplified view of the stator housing with some of the relevant design parameters. The corresponding design stream (parameters flow) is illustrated in Fig. 8. 


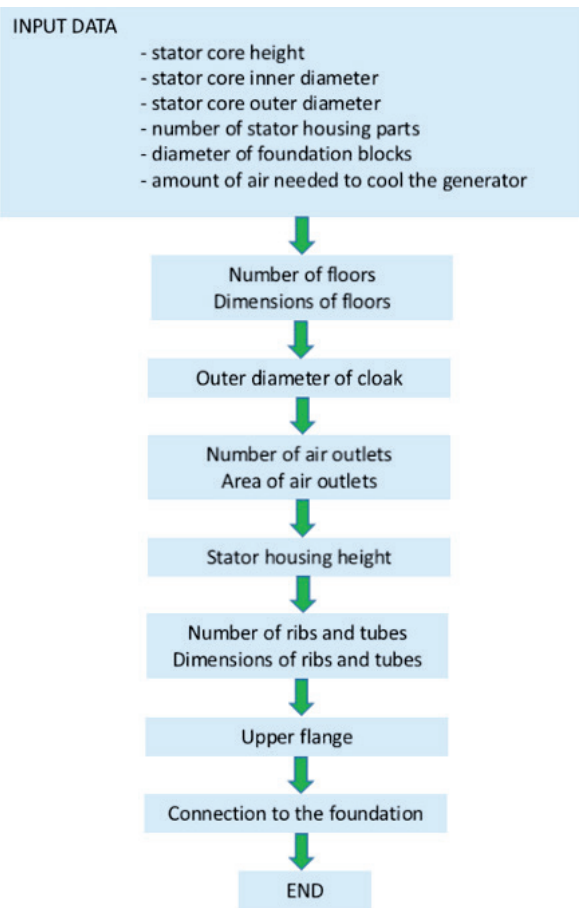

Figure 8 Stator housing design stream

Fig. 8 illustrates the precedence of design parameters: to design the stator housing the information about stator core height, height of stator end winding, stator core inner diameter, stator core outer diameter and number of stator parts, diameter of foundation blocks and amount of air needed to cool the generator are needed in order to define number of floors and floors dimensions. Afterwards the designer will determine outer diameter of cloak, number of air outlets and needed area of air outlet. In the next step stator housing height, number of ribs and tubes, dimensions of ribs and tubes, including the upper flange form and dimensions are determined. In the last phase connections to the foundation blocks are defined.

In a similar manner the design parameter flows are defined for all the components of the hydrogenerator.

\subsection{DSM of Vertical Generator Design Parameters}

The first part of unpartitioned DSM matrix comprises main electrical parameters (nominal voltage, power, frequency, power factor, nominal speed, principal dimensions, materials etc.), and this part of the matrix is filled-in and verified by electrical engineering experts. The rest of matrix contains all other relevant design parameters of main generator parts like stator housing, stator core, upper bracket, lower bracket, shaft, rotor spider, rotor rim, poles, the upper cover, the lower cover, fans, and braking device. Except that, DSM contains parameters used in mechanical calculations like upper bracket stiffness, lower bracket stiffness, the torsional stiffness of rotor spider, critical speed, forces on the upper bracket, forces on lower bracket, braking time, braking pressure, the pressure needed for lifting of the rotor, etc. Although, as said earlier, the design experts composed the DSM matrix, due to its complexity it is still under verification. Full unpartitioned DSM matrix before final check is shown in Fig. 9. Turbine design parameters are not in the scope of this work, just generator's main parts: shaft, rotor spider, rotor rim, poles, fans, bearing collars, brackets, stator housing, stator core, stator winding, bearings, etc., and electrical parameters which influence the design process. Necessary turbine information is taken into analysis through one field called turbine data comprising a set of turbine parameters needed for generator design. In addition, a field with customer requirements is also incorporated uniting the set of relevant parameters used for generator design.

After partitioning (reordering rows and columns) of DSM matrix, we got almost straightforward flow with few blocks of coupled parameters. Partitioned DSM is illustrated in Fig. 10. We used free program for partitioning using Reachability Matrix Method DSM_Program-v2.1 from DSMweb.org. DSM of design parameters in the current, working version includes 221 parameters. Fig. 10 represents the partitioned matrix; for better visualisation all interactions are marked with black dots. It should be noted that design experts gather these matrix data, so it is based on the knowledge and experience more than on the prescribed procedures which cover only the standard situations.

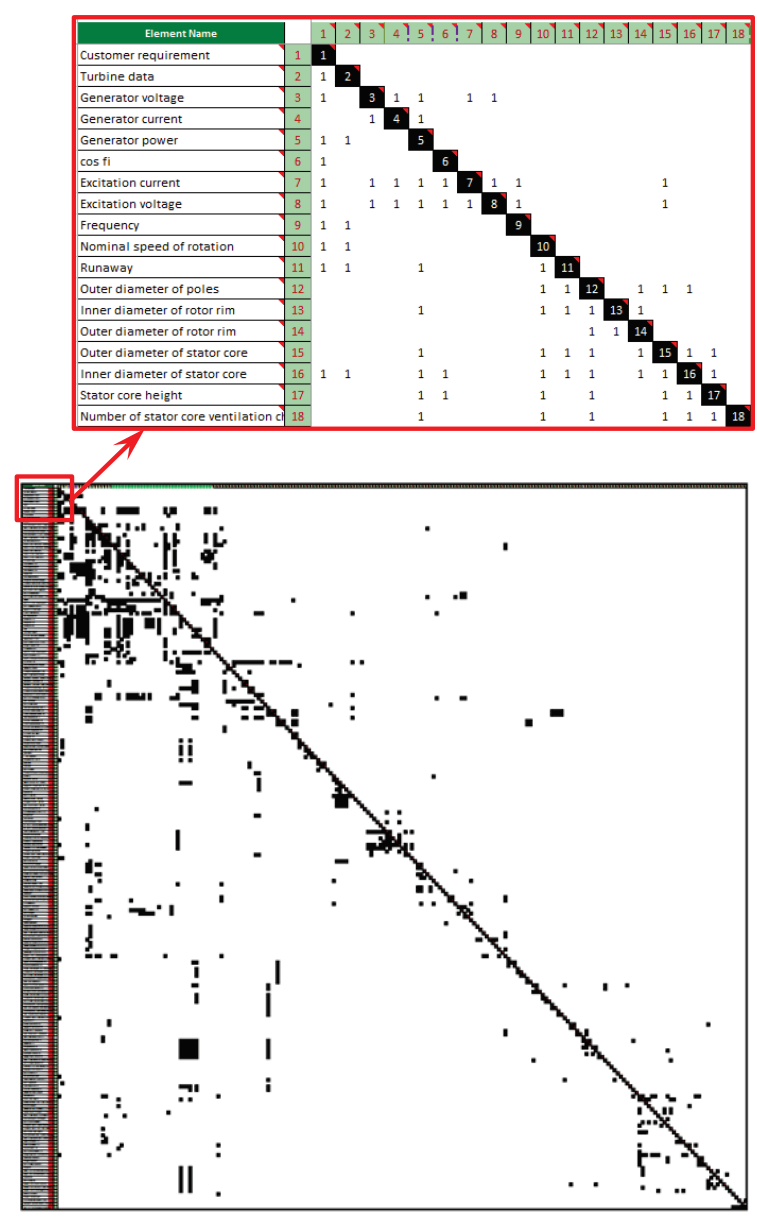

Figure 9 Unpartitioned DSM of main design parameters

Partitioning of the matrix shows that following the customer demands and turbine data, the concern is given to the materials which are predefined (because they are the same in almost all generators) and then electrical design goes in the first phase at the beginning of the project. In that electrical design, there are few construction design parameters which should be checked preliminarily to avoid later iterations. With complete electrical design, a mechanical design could be done almost straightforward 
with minimal iterations. The presented DSM of vertical hydrogenerator is further expanded to model relationships between parameters and engineering drawings, calculations, etc.

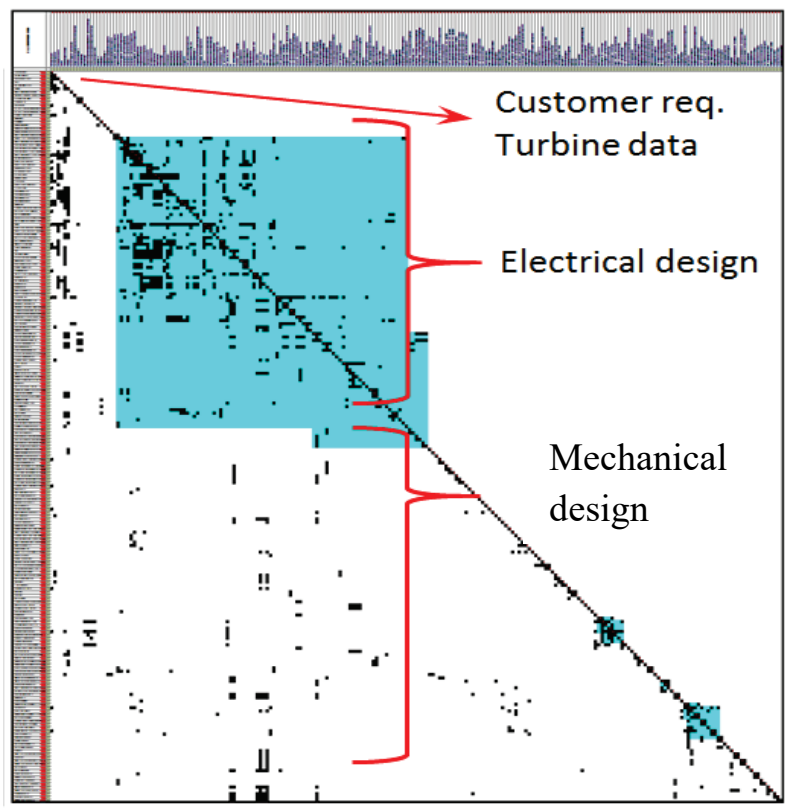

Figure 10 Partitioned DSM of main design parameters

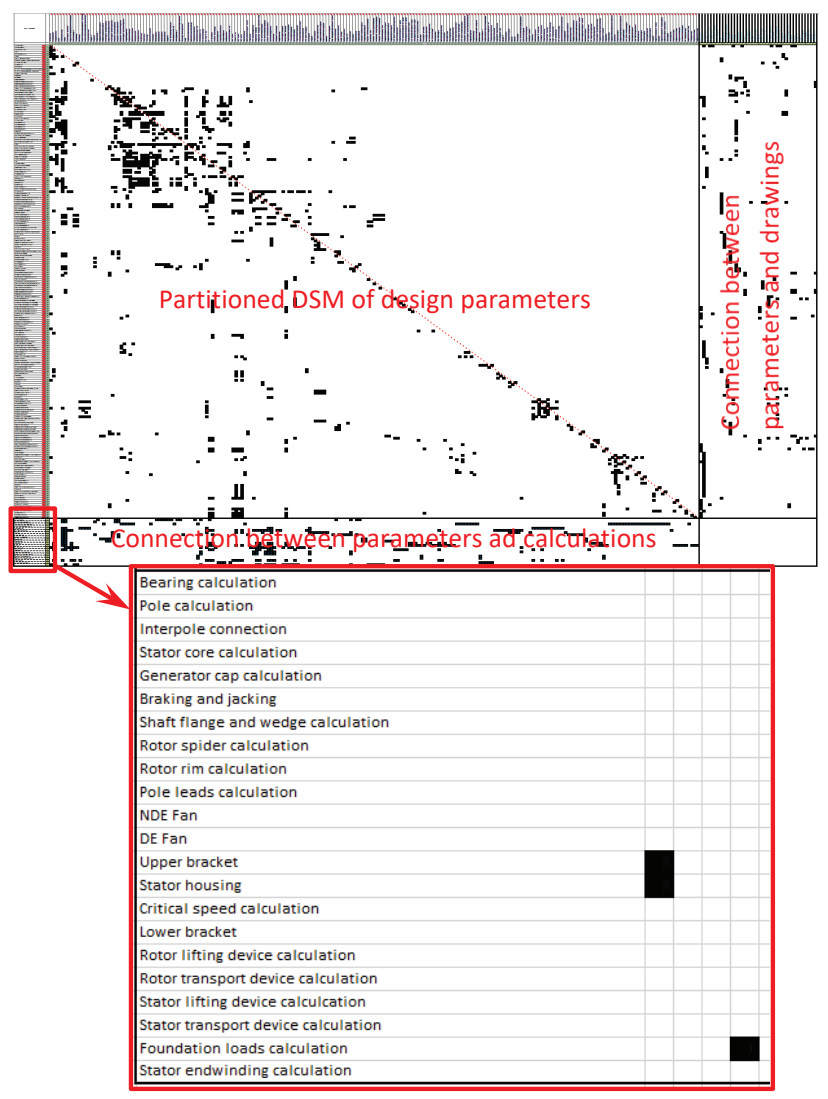

Figure 11 MDM of parameters - calculations - drawings

\subsection{MDM Matrix}

To model relationships between components, design parameters and engineering calculations and engineering drawings MDM turned out as the best and easiest solution from which it will be possible to visualise interactions between parameters, calculations and drawings. In Fig. 11 such MDM matrix is illustrated with major areas explained below.

Upper left submatrix is partitioned DSM of design parameters, lower left submatrix is matrix of interdependence between parameters and calculations (calculations are shown in Fig. 3), upper right submatrix represents interdependence between parameters and drawings (shown in Fig. 2), and lower right submatrix should represent interconnection of drawings and calculations (it is not used for now).

\subsection{Visualizing the Relationships and Interdependencies}

Although the MDM is still in the phase of development and verification, it was apparent that a tool for visualisation of relationships and dependencies needs to be developed. For the research purposes, an application has been developed.

Based on the designers input the application graphically selected design parameter and all affected parameters, drawings and calculations, and if needed lists the specification and the order of dependencies.

Purpose of this application is to visualise the propagation of design process and design changes by allowing a user to enter parameter which is or should be changed, and a program should write down and visualise all affected parameters, calculations and drawings which should be updated or changed. At the moment the application is developed with the assumption that there is no reverse iteration (similar as it is done in banding algorithm), i.e. assumption is that when someone wants to make change on some parameter, affected parameters already have some value (they are already determined), and there will not be necessary to get that parameter again iteratively. The time will show whether that assumption is ok or not, and besides, it will take years to clear doubts in interactions and to find interactions which are not visible at the moment.

Although the application is intended primarily for electrical engineers, it is useful for all involved in the development process, the managers and the designers could use and track changes in design, being aware of the consequences of design parameter changes. With such a tool decision making should be easier and less error-prone.

To demonstrate how the program works, the following figure shows a little example with MDM in which are randomly selected interactions. Submatrices are similar as it is written before, upper left submatrix is DSM of parameters, lower left submatrix is matrix of interdependence between parameters and calculations, upper right submatrix represents interdependence between parameters and drawings, and lower right submatrix should represent interdependence of drawings and calculations, but it is not used in program for the time being.

When user selects first parameter, changing of the first parameter affects par. 2, par. 5 and par. 6. Parameter 2 affects par. 3 and par. 7, par. 3 also affects par. 6, par. 5 affects par. 6 and par. 8. All mentioned affected parameters are highlighted in red colour. If we must change parameters $1,2,3,5,6,7,8$, they affect calculation 1 , calculation 2 , calculation 5, calculation 6 and calculation 8 (shown with green colour), except that they will affect drawing 1 , 
drawing 2, drawing 3, drawing 5 and drawing 9 (shown with yellow colour).

Further a need to optimise calculation and drawing paths in that way that they follow an optimal order of partitioned parameters occurred during testing. This VBA program will be updated in later version, to additionally reorder drawing columns that way that first drawing from the left is one which could be done first (following dependencies between parameters and drawings), on lower example, first drawing will be drawing 4 , and then goes drawing 6 , drawing 8 , drawing 1 , drawing 5, drawing 9, etc. The same procedure will be integrated to optimise calculations stream. That way, this MDM will give the optimal solution to minimise iterations during designing of a generator or something else.

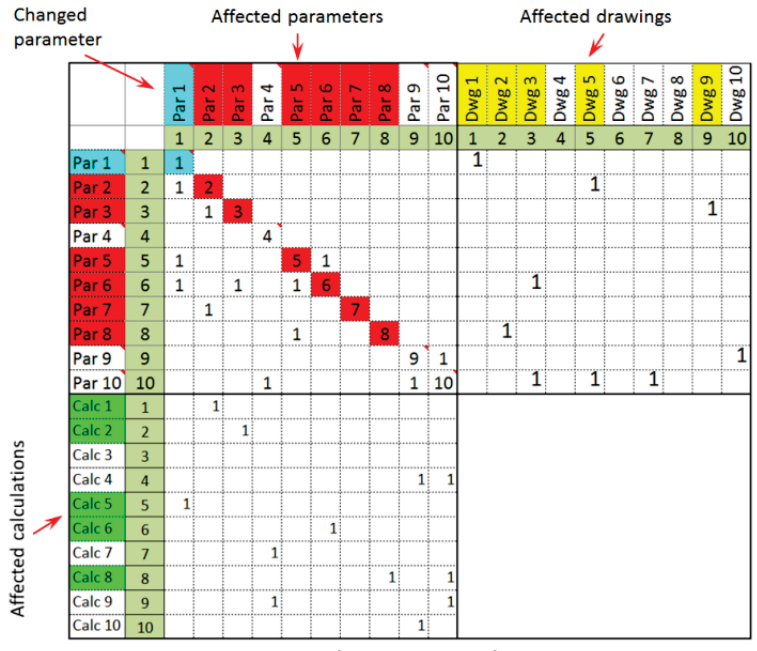

Figure 12 Example of VBA program for MDM matrix

The developed programme based tool enables instant visualisation of relationships in the MDM. Based on the selected parameter, all the affected parameters will be highlighted, including related calculations and drawings as it can be seen in Fig. 13. All affected parameters are coloured with red colour, while affected calculations are coloured with green, and drawings will be coloured with yellow.

There is still one open problem, which is not visible in the same moment, drawings optimal order (after partitioning matrix of all drawings), and calculations optimal order (after partitioning DSM of all calculations) could have different optimal path than path given with design parameters. That makes a new question "is it possible to minimise rework or time needed to finish the project from beginning to the end (with all mentioned domains)?". Except that, will matrix of interdependences of calculations and drawings show the same affected calculations and drawings?

Those questions should be considered in future but for the time being an answer for the first question could not be obtained either with these DSM or with MDM matrix.

\section{CONCLUSION}

Although the initial project goal to develop a product DSM enriched with the design parameters of the vertical generator, the DSM analysis opened new possibilities which at the end led to MDM matrix and later to the VBA program for MDM matrix.

MDM matrix with VBA program was developed with an intention to help designers, managers and others, to help them track changes during a design process. To help designers and others in making decisions (is it possible to change some parameter or not, and what that change means), and at the end to help a user to know who should be warned in case of making the change of some parameter.

The MDM matrix and the VBA program tool are developed to help designers, managers and other process stakeholders, to help them track changes during the design process. To help designers and others in making decisions (is it possible to change some parameter or not, and what that change means), and at the end to help the user to know who should be warned during the changes made to design parameters. MDM of this size has very nice visualisation opportunities as it can be seen in Fig. 13.

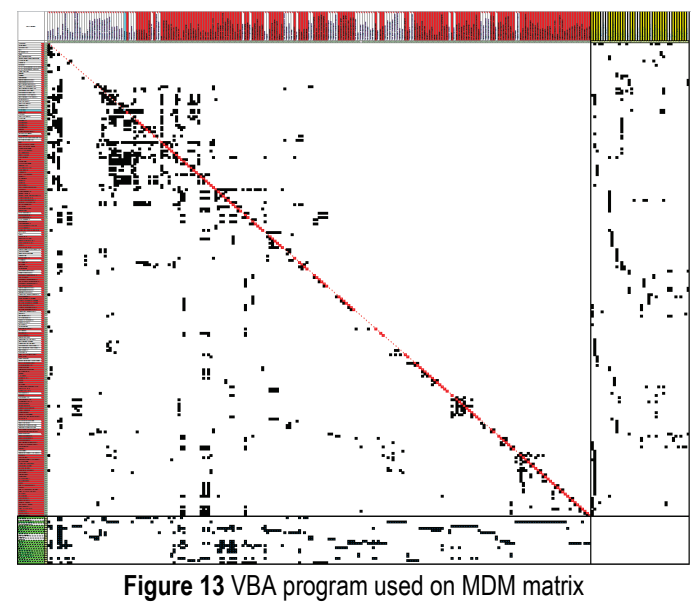

That visualisation helps the user to understand the problem better and intuitively. The proposed colour scheme should help users to easily track how changed parameter affects other parameters and enable quick access to relevant information from which it could be concluded if it is smart to change some parameter, and how much rework should be expected. Future work will refer to updating of VBA program to give an optimal order of calculations and drawings and finishing with checks of DSM and MDM, making updates on relations in DSM and MDM. It will take many years to get the fully usable program. The implementation of the system in a daily routine work is still to be accomplished within the company.

\section{REFERENCES}

[1] Arnarsson, I. O., Gustavsson, E., Malmqvist, J., \& Jirstrand, M. (2018). Analysis of Engineering Change Requests Using Markov Chains. Proceedings of the DESIGN $201815^{\text {th }}$ International Design Conference, Dubrovnik, Croatia. https://doi.org/10.21278/idc.2018.0314

[2] Browning, T. (2001). Applying the Design Structure Matrix to System Decomposition and Integration Problems: A review and New Directions. IEEE, 48(3). https://doi.org/10.1109/17.946528

[3] Browning, T. R. (2009). The Many Views of a Process: Towards a Process Architecture Framework for Product Development Processes. Systems Engineering, 12, 69-90. 
https://doi.org/10.1002/sys.20109

[4] Browning, T. (2016). Design Structure Matrix Extensions and Innovations: A Survey and New Opportunities. Transactions on Engineering Management, 63(1). https://doi.org/10.1109/TEM.2015.2491283

[5] Danilovic, M. \& Browning, T. (2007). Managing Complex Product Development Projects with Design Structure Matrices and Domain Mapping Matrices. International Journal of Project Management, 25(3), 300-314. https://doi.org/10.1016/j.jproman.2006.11.003

[6] Eppinger, S. \& Browning, T. (2012). Design Structure Matrix Methods and Applications, MIT press, London, England. https://doi.org/10.7551/mitpress/8896.001.0001

[7] Eppinger, S. \& Salminen, V. (2001). Patterns of product development interactions. Proceedings of International Conference on Engineering Design, ICED'01, August 2001.

[8] Erixon, G. (1998). Modular function deployment: a method for product modularisation. PhD Thesis, Dept. of Manufacturing systems, KTH Royal Institute of Technology, Stockholm, Sweden.

[9] Farooq A., Araújo A., Tavares S. M. O., \& Nóvoa H. T. (2013). Evaluation of a non-conformity matrix complexity using components modularity metrics. $15^{\text {th }}$ International DSM Conference. https://doi.org/10.3139/9783446437937.003

[10] Halonen, N., Juuti, T., \& Luostarinen, P. (2012). DSM made easy. International design conference Dubrovnik, Croatia.

[11] Göhlich, D., Hildebrand, S., \& Schellert, D. D. (2018). Augmented DSM Sequencing to Support Product Development Planning. Proceedings of the DESIGN 2018 $15^{\text {th }}$ International Design Conference, Dubrovnik, Croatia. https://doi.org/10.21278/idc.2018.0434

[12] Lindemann, U. (2009). Structural Complexity Management: An Approach for the Field of Product Design, Springer, Berlin, Heidelberg.

[13] Maurer, M. (2007). Structural Awareness in Complex Product Design, Dissertation, München.

[14] Maurer, M. (2017). Complexity Management in Engineering Design - a Primer, Springer-Verlag, München, Germany. https://doi.org/10.1007/978-3-662-53448-9

[15] Morelli, D., Eppinger, S., \& Rosaline, G. (1995). Predicting Technical Communication in product Development Organizations. IEEE Transactions on Engineering Management, 42(3), 215-222. https://doi.org/10.1109/17.403739

[16] Olmez, H., Hassannezhad, M., Ball, N., \& Clarkson, P. J. (2018). Modelling Change with an Integrated Approach to Manufacturing System Design. Proceedings of the DESIGN $201815^{\text {th }}$ International Design Conference, Dubrovnik, Croatia. https://doi.org/10.21278/idc.2018.0401

[17] Steward, D. V. (1981). Systems Analysis and Management, New York: PBI.

[18] Steward, D. V. (1981). The Design Structure System: A Method for Managing the Design of Complex Systems. IEEE Trans. on Eng. Mgmt., 28, 71-74. https://doi.org/10.1109/TEM.1981.6448589

[19] Talić, A. (2013). DSM Method - design structure matrix. $17^{\text {th }}$ International Research Conference, Istanbul, Turkey.

[20] Triplat, I., Vukšić, M., \& Marjanović, D. (2016). Application of DSM to mechanical calculations of vertical hydro generator. International Design Conference Cavtat, Croatia.
Contact information:

Ivan TRIPLAT, mag. ing. mech.

KONČAR Generators and Motors Inc

Fallerovo šetalište 22, 10000 Zagreb, Croatia

itriplat@koncar-gim.hr

Dr. Sc. Dorian MARJANOVIĆ, dipl. ing.

Faculty of Mechanical Engineering and Naval Architecture Ivana Lučića 5, 10000 Zagreb, Croatia dorian.marjanovic@fsb.hr

\section{Lucijan STAMAĆ, dipl. ing.}

Faculty of Mechanical Engineering and Naval Architecture Ivana Lučića 5, 10000 Zagreb, Croatia

lucijan.stamac@fsb.hr 